\title{
Patchwork organization of the yeast plasma membrane into numerous coexisting domains
}

\section{Felix Spira, Nikola S. Mueller, Gisela Beck, Philipp von Olshausen, Joachim Beig \& Roland Wedlich-Söldner}

Nat. Cell Biol. 14, 640-648 (2012); published online 29 April 2012

In the version of this article initially published, the figure legends lacked an acknowledgement that the cell shown in Fig. $7 \mathrm{~b}$ is identical to that in Fig. 8a (top right). The colocalization experiments were performed with controlled acquisition protocol, temperature, culture density and genetic backgrounds. The experimental conditions for Fig. $7 \mathrm{~b}$ were therefore identical to those for Fig. $8 \mathrm{a}$ (top right). The same cell is shown in both panels as a point of comparison to illustrate the degree of protein colocalization. 